\title{
- BEAUTY IN DISGUISE - THE PHYSICS BEHIND THE POWER GRID
}

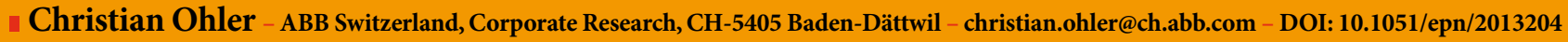

The power system is built from four main components - overhead lines, transformers, generators, and circuit breakers. Physics determines their shape, and by looking at their shape, we learn how power grids work.

$\checkmark$ High voltage lines near Uckerath, Germany (Photo: Michael Ploss)

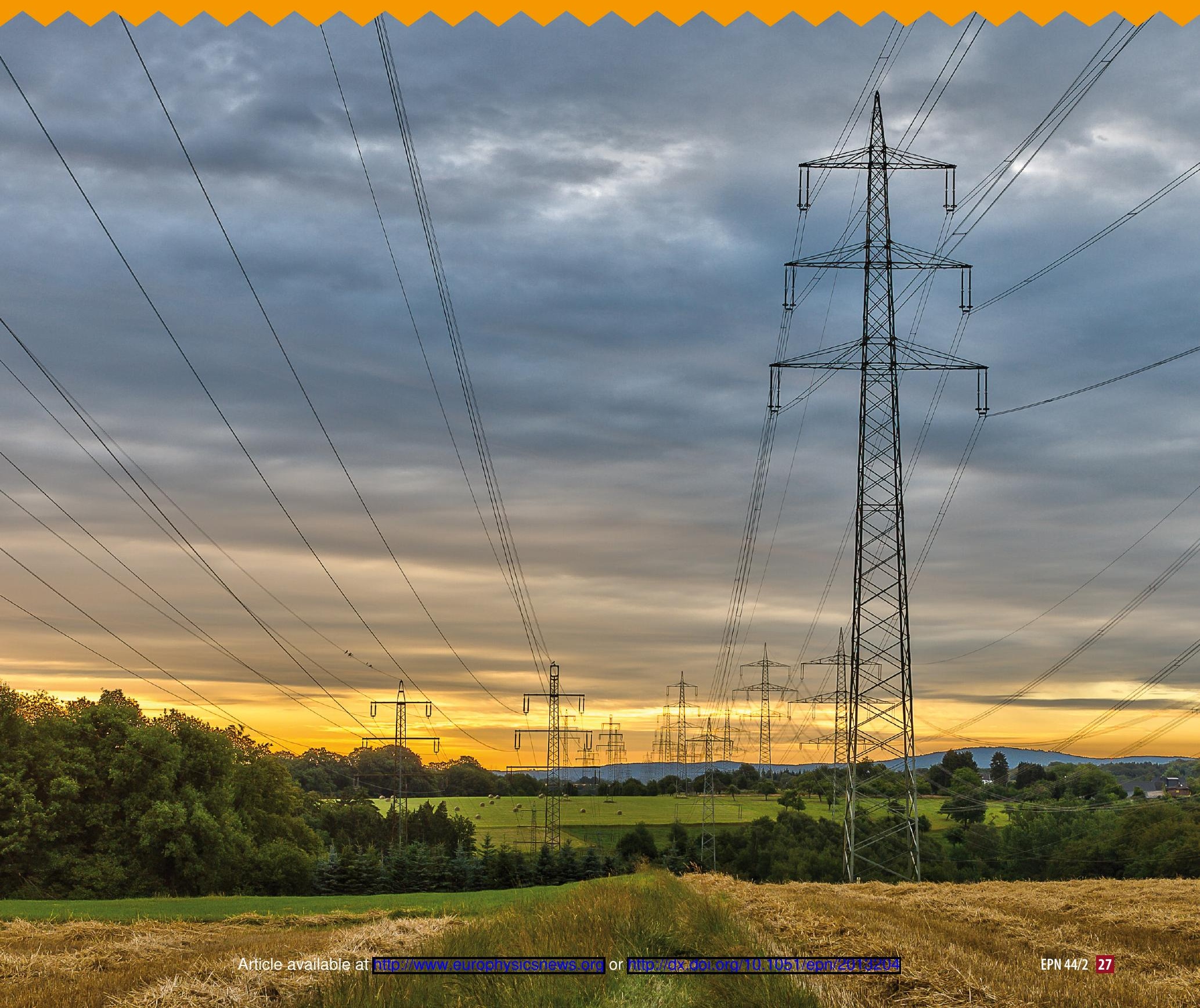


v FIG. 1. Silicone outdoor insulation

from a cable termination. The

shape with its multiple umbrellas,

the sheds, and the hydrophobic

surface prevent the formation of a continuous water

film. The suspension insulators on high voltage overhead line towers are of a similar

design, but with smaller diameter. (Photo: C. Ohler)
$\mathrm{N}$ ext time you pass by a tower of a high voltage overhead line, please look at it with fresh eyes. Isn't it beautiful in the way its form follows its function? Do you remember why it is a tower at all? It is because of the high voltage that needs to be kept away from passing people. And why does the voltage need to be so high? It is because we need to save on current. Resistive losses go with the square of the current. Transmitted power is the product of current and voltage. The higher the voltage, the lower the current and the less power per distance we lose.

A line tower is a miniature Eiffel tower. The lattice structure minimizes the amount of structural steel for a given mechanical load and height and lets the wind pass through without offering resistance.

Another interesting shape is that of the surface of the cylindrical solid insulators holding the 'live' parts in place (see Fig. 1). Rain water would form an electrical short to the tower structure on ground potential. It is prevented from forming a continuous path along the insulator surface by a number of small umbrellas, the sheds. Sheds force rain water to drip along the insulator surface and not to trickle. Sheds are the earmarks of outdoor insulation and by counting the number of sheds per insulator you can guess the voltage level of an overhead line. The only thing you need to know is that a single shed can stand a voltage difference of 10 kilovolts or so.

The conductors of high-voltage lines often come in bundles of two or four per phase. A bundle of conductors approximates, with some effort of the imagination, a hollow cylinder that lets the wind blow through. Such a cylinder has a higher surface area than a massive conductor of the same cross-section, and the electric field at the conductor surfaces is small enough to avoid the ionisation of the adjacent air - a corona discharge - that would create losses and make noise. The suppression of corona discharges is not perfect, as you can tell from the sizzling of high voltage lines in humid weather conditions.

The second reason for bundles is the skin effect. The current density of an alternating current is pushed towards the outer surface of any conductor. Rather than wasting aluminium in the centre, it is provided at the outside where the current prefers to flow. Why aluminium and not copper? Sure, copper has a better electrical conductivity by a factor of 1.6, but it is heavier by a factor of more than 3 and more expensive by a factor of 4 . So most high-voltage lines are made of steel-reinforced aluminium conductors. The larger effective diameter of the current density reduces also the parasitic self-inductance of the overhead line. This might appear to be a marginal detail, but the fact that lines, transformers, and many of the motor loads come with parasitic inductances is a severe constraint of power system operation. The energy that flows periodically into and out of the magnetic field of parasitic inductances is named "reactive" power, and it has to be supplied by circuit elements of opposite, capacitive, kind. The capability to behave like a capacitor is one of the merits of synchronous generators (see below).

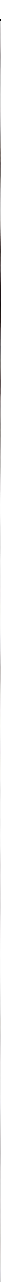




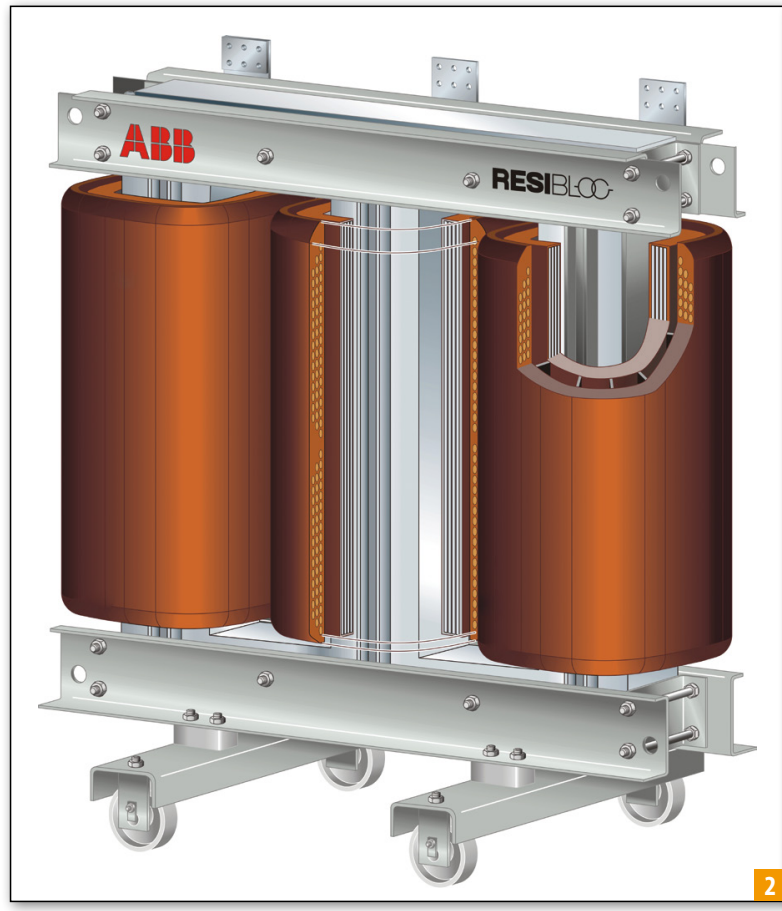

\section{Real transformers don't look like the one in the textbook}

A child observing high voltage overhead lines during a car trip will soon notice that the conductor bundles come in sets of three. They herald the three-phase nature of the power system. Three is the smallest number of phases that allows for a constant, time-independent supply of power from alternating, time-varying current and voltages. Three sinusoidal currents of the same magnitude and displaced in time by one third of the period sum up to zero at any time. As a consequence, the return conductors of the three phases can be omitted. Compared to single phase systems, three-phase systems carry the same power with half of the conductor mass.

Transformers work only for alternating current. They step up the voltages from the power plant so that electric power can be transmitted over long distances, and they cascade the voltage down for distribution and consumption of power. Transformers work through the combined action of Ampere's law - all electric currents come with a surrounding magnetic field - and Faraday's law - time varying magnetic fields induce electric fields and hence currents in conductors.

Real transformers don't look like those in physics textbooks. If they would, their leakage inductance would be terrible. Instead, for each phase, high voltage and low voltage winding are put coaxially around a stack of thin laminated layers of core steel (fig. 2). The two windings should be as close as possible to each other because the magnetic flux is only imperfectly contained in the core. Nature has provided us with metals that have higher electrical conductivity than insulators by twenty-four orders of magnitude, but she has been less generous in orders of magnitudes for magnetic

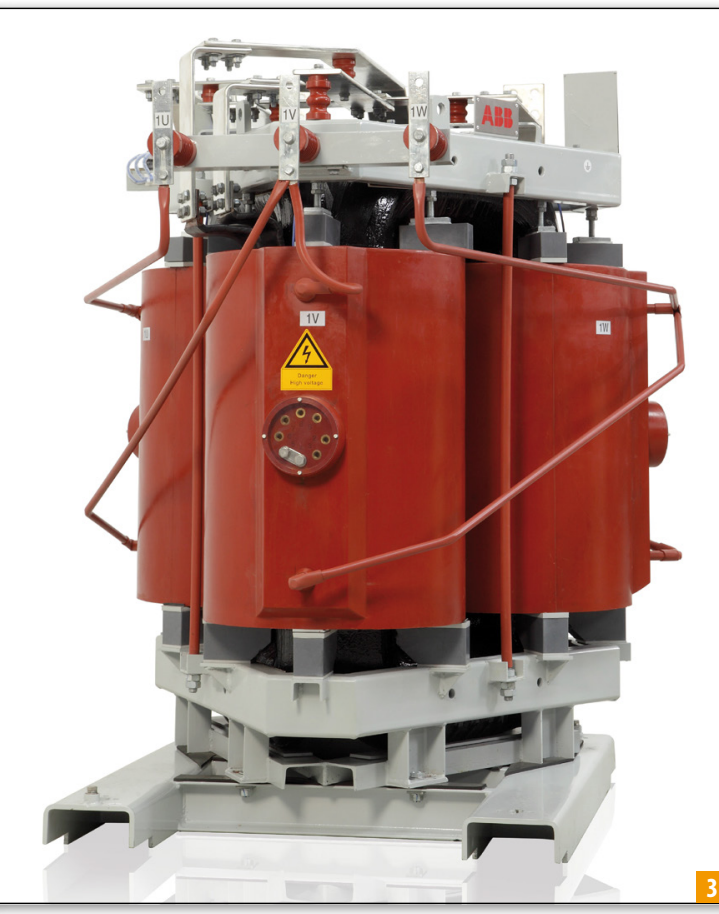

permeability. It is left as a thought experiment to the reader how power systems would look like if it was the other way around.

Three-phase power transformers use the same trick for the magnetic flux that three-phase overhead lines use for the electric current. The sum of the magnetic return fluxes cancels, and there is no return leg for any of the phases. A three-phase transformer transforms twice as
4 FIG. 2.

Cut-away view of a dry distribution transformer. For each of the three phases, high voltage and low voltage winding are put coaxially around a core of laminated stee plates. (Source: ABB)

4 FIG. 3.

Dry distribution transformer with triangular core for minimal space and material usage. (Source: ABB)

vFIG. 4.

Rotor of a synchronous generator with two poles. The coil is wound along the cylinder axis. It is supplied with a direct current for creating the rotating magnetic field that induces oscillating currents in the stator windings. (Source: ABB)

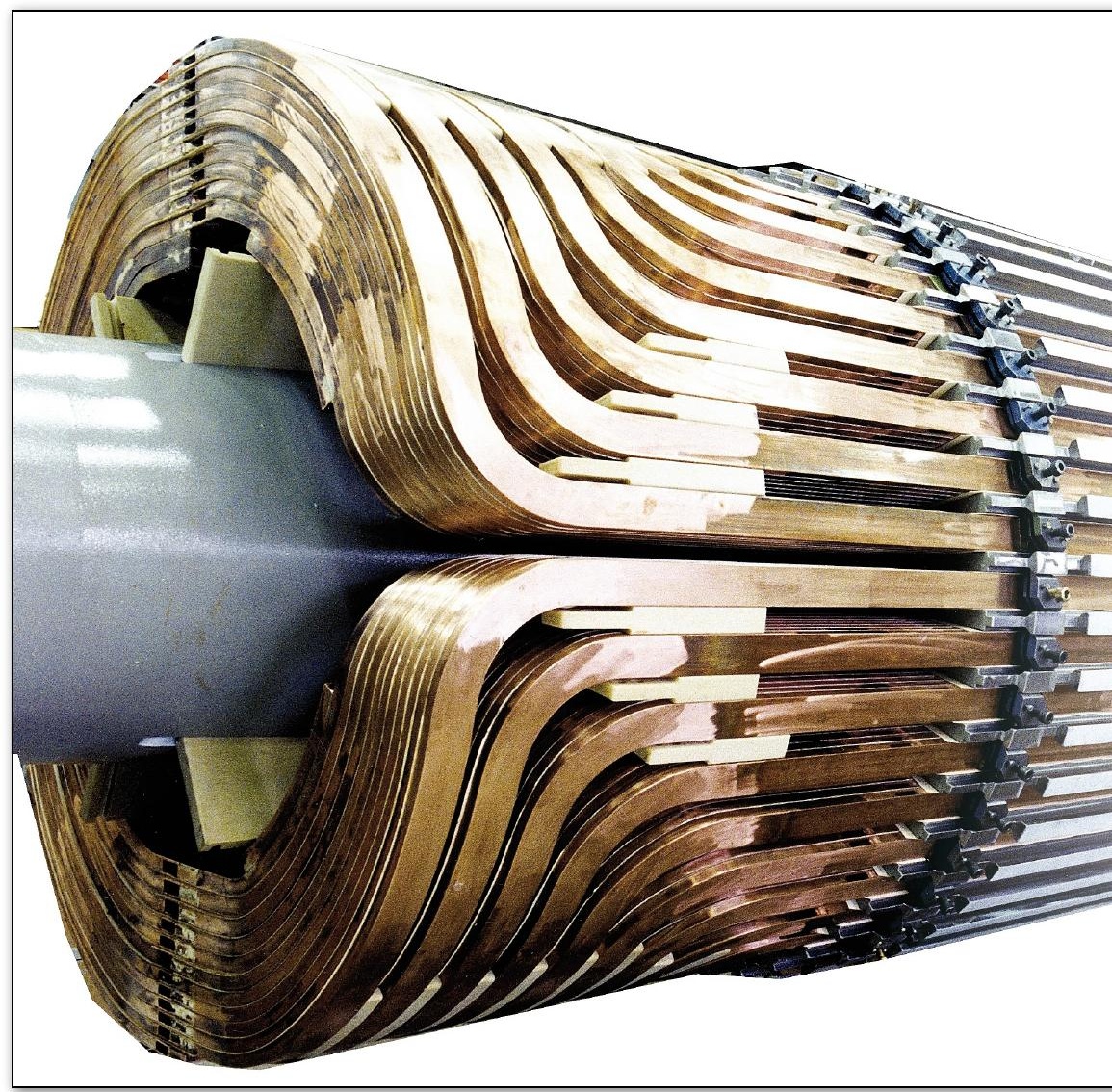


much power as three single-phase transformers of the same core size would.

The shape of power transformers is the result of the intention to wind two coils around each other and around a core cylinder, while maintaining sufficient insulation distance in between, and joining the three legs of the phases with as little connecting yoke as possible. The cross-section of the steel cores and the steel yoke is just large enough to avoid magnetic saturation. A particularly illustrative layout of the power transformer is the triangular transformer shown in fig. 3 .

\section{Winding coils "the wrong way"}

Most of the energy fed into the power grid comes from synchronous generators. They convert the mechanical power of the power plant into electrical power. Being made of coils, it is worth noting that synchronous generators can provide reactive power, i.e., behave as a capacitor.

The shape of a generator follows from the need to wind coils around a cylinder "in the wrong way", alongside the cylinder's axis. This is to convert a steady mechanical rotation into a rotating magnetic field that induces three currents that periodically oscillate. The winding of the rotor (fig. 4) is fed with a direct current and, driven from the mechanical energy of the connected turbine, creates a rotating magnetic field just like a rotating permanent magnet would do. At each rotation, the rotor field passes by the stator windings (fig. 5) that are connected such that they form three coils displaced by 120 degrees along the circumference of the cylinder. These three coils are connected to the three-phase power grid, and the magnetic rotor field induces periodically a current in each of them. Depending on the angle of the rotating rotor relative to the voltage of the power grid rotating with the same frequency, the machine will either push active power into the grid acting like a generator, or consume active power from the grid acting like a motor.

\section{Circuit breakers as judokas}

If the synchronous generators are the kings of the power grids, circuit breakers are their knights, always ready for a fight, but rarely fighting. Imagine for a moment the vast network of conductors extending over the continent and linking all the corners of Europe. Imagine the likelihood of a short circuit somewhere. If there weren't an element for segmenting the healthy from the faulty parts of the network, then "good night!" indeed.

Circuit breakers are electrical switches designed to protect a power circuit from damage caused by short circuits. They are ideal conductors in the closed position, ideal insulators in the open position, and capable of interrupting short circuits that may be orders of magnitude larger than the normal current they carry.

The outer shape of a simple high-voltage circuit breaker follows in a straightforward way from its function (fig. 6). For each of the three phases, it is a linear, cylindrical segment - the interruption chamber - inserted between the terminals of an incoming and outgoing conductor.

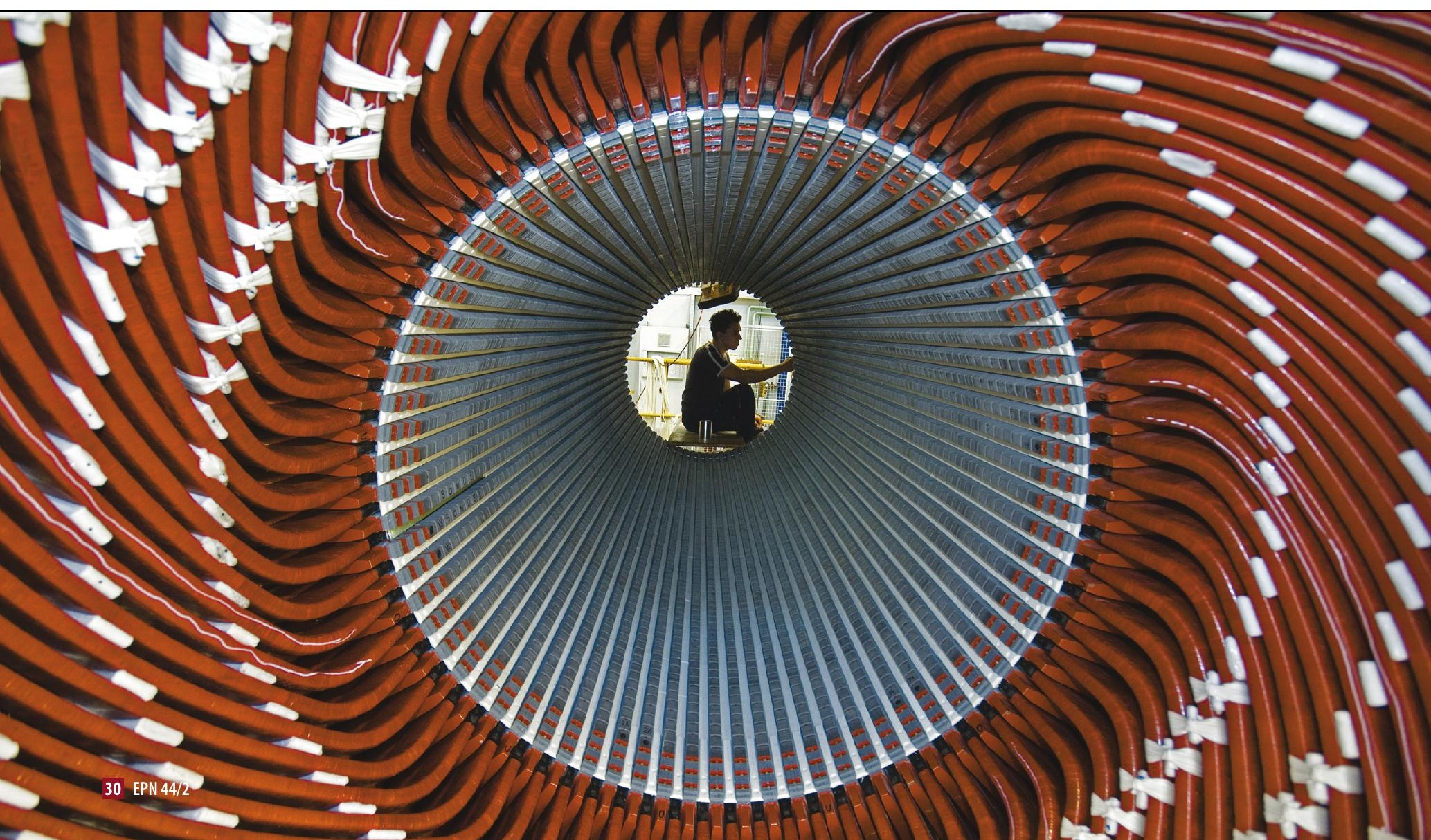


In open position, one of the terminals may be on system voltage and the other may be on ground. Thus the outside of the interruption chamber needs to have an outdoor insulation with sheds to avoid an outside insulation failure. In normal operation both conductor sides are at high voltage; in consequence, the whole interruption chamber is supported by an outdoor insulator of the same design.

Circuit breakers fight in the way of a judoka. They exploit part of the energy inherent in the short-circuit to tame the short-circuit. Their inner life is complex and based on plasma physics and hot gas dynamics (see box).

\section{Please greet the line towers}

The power grid was there when we were born. We tend to forget what a beautiful achievement of our society it is. The synchronous grid of continental Europe is the largest in the world by installed power, and the average outage time of a customer is 15 minutes per year. The grid is on $99.997 \%$ of the time.

With curious eyes you will see transformers and circuit breakers through the fence of outdoor substations. But the only components of the grid visible to the general public are the overhead lines and their towers, the servants of our power system. A surprising number of things about the power grid can be learned from looking at a line tower. Next time you cross one of these humble servants, please greet him with the physicist's respect that he deserves.

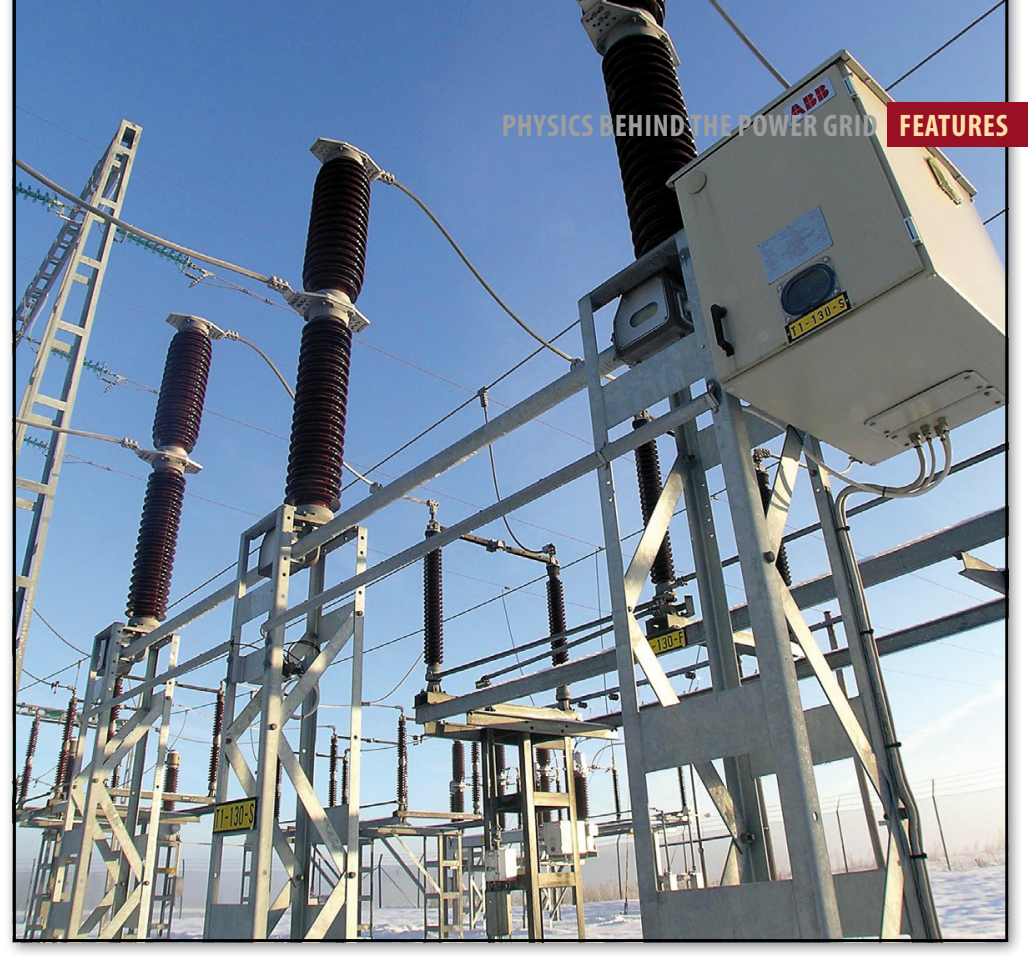

\section{About the Author}

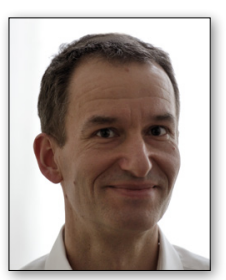

Christian Ohler is leading the department "Power products and sensors" at ABB's Corporate Research Centre in Switzerland. He has a master degree and $\mathrm{PhD}$ in Physics from RWTH Aachen. ABB is a global company in power and automation technologies and employs 145,000 people in 100 countries.

\section{References}

[1] N. Mohan, Electric power systems: a first course, Wiley, New York, 2012.

[2] A. Küchler, High voltage engineering: fundamentals, technology, applications, Springer, Berlin, 2013.

\section{BOX}

Figure 7 shows a cut through the cylindrical interruption chamber of a high-voltage circuit breaker at different moments of action as a short circuit is interrupted. Letters refer to the elements of the circuit breaker, numbers to instants in time. In closed position, both kind of contacts, the main contacts (A) and the "arcing" contacts (B), are closed and the main contacts carry most of the current with low losses. Upon the detection of a short circuit by a current sensor that is a separate device, both contacts move, first separating the main contacts and transferring the current to the arcing contacts. When the arcing contacts separate (1), a hot plasma arc is drawn in the gap, and the short circuit current continues to flow through it, providing ample heat so that the plasma remains conductive. During the high-current phase (2), some part of the energy is used to build up pressure in dedicated volumes (D). Shortly before current zero (3) the gas flow reverses and cools the arc convectively as it shrinks with the declining current. At current zero (4), with sufficient cooling flow provided, the current is interrupted, and the gap between the contacts (and also $\triangle$ FIG. 6 .

Three-phase high-voltage live tank circuit breaker.

(Source: ABB)

V FIG. 7: A cut through the cylindrical interruption chamber of a high voltage circuit breaker at different moments during current interruption. The temperatures of the interrupting gas, sulphur hexafluoride, are indicated by the color. Light blue/grey: room temperature gas; red: hot plasma, up to $20^{\prime} 000^{\circ} \mathrm{C}$; yellow: intermediate. The green shape is an insulating component guiding the hot plasma flow. The total time of the interruption process shown here is about 20 milliseconds.

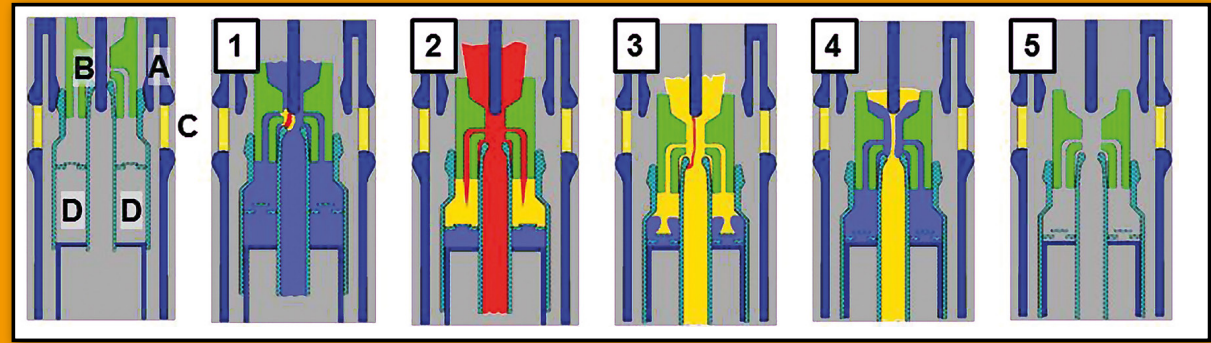

the chamber insulator, C) has to sustain immediately the recovery voltage (5). In a successful interruption, the dielectric strength of the gas recovers faster than the recovery voltage of the network rises. Otherwise, in the case of a failed interruption, there will be a breakdown, and the arc will reignite. 